\title{
Interaction between Seizure and Theta Rhythm
}

\author{
Zakaria I. Nanobashvili1,2, Irine G. Bilanishvili1,3, Maia D. Barbakadze1, Tamar Z. Gaixarashvili3, \\ Salome A. Dolidze ${ }^{3}$, Ketevan E. Khujadze ${ }^{3}$, Levan X. Nachkebia ${ }^{3}$, Nadejda A. Khizanishvili ${ }^{1}$
}

${ }^{1}$ Department of Neurophysiology, I. Beritashvili Center of Experimental Biomedicine, Tbilisi, Georgia

${ }^{2}$ International Caucasus University, Tbilisi, Georgia

${ }^{3}$ P. Shotadze Tbilisi Medical Academy, Tbilisi, Georgia

Email: zaqariananobashvili@gmail.com

How to cite this paper: Nanobashvili, Z.I., Bilanishvili, I.G., Barbakadze, M.D., Gaixarashvili, T.Z., Dolidze, S.A., Khujadze, K.E., Nachkebia, L.X. and Khizanishvili, N.A. (2020) Interaction between Seizure and Theta Rhythm. Journal of Behavioral and Brain Science, 10, 18-28.

https://doi.org/10.4236/jbbs.2020.101002

Received: November 27, 2019

Accepted: January 3, 2020

Published: January 6, 2020

Copyright $\odot 2020$ by author(s) and Scientific Research Publishing Inc. This work is licensed under the Creative Commons Attribution International License (CC BY 4.0).

http://creativecommons.org/licenses/by/4.0/

\begin{abstract}
Recently it was shown by us that combined stimulation of hippocampus and dorsomedial hypothalamus resulted in suppression of the electroencephalographic seizure reactions and, respectively, manifestations of behavioral seizures reduced. It is expected, that augmentation of inhibitory processes in hippocampal neurons in the course of dorsomedial hypothalamus stimulation can trigger mechanisms preventing the development of epileptiform activity. Because of two important characteristics of the hippocampus-theta rhythm and epileptogenesis-these appear to be interrelated in respect of their cellular substrates, and as far as theta rhythm may modulate hippocampal excitability, a study of the functional relationship between theta rhythm and seizure activity was endeavored. The purpose of this study is to test this proposal by determining the effects on seizures of induction or suppression of hippocampal theta activity. Our findings show that: 1) against background of strong unusual sound stimulation (in our case-sound) blockade of local seizure reactions induced by hippocampal stimulation occurred;2) the frequency of hippocampal interictal epileptiform dischargers increased with the transition from the awake state to drowsiness and a slow-wave sleep phase. After the animal came from slow-wave sleep to paradoxical sleep, epileptiform activity completely disappeared; 3 ) at threshold stimulation of hypothalamus when electrohippocampogram shows augmentation of the theta rhythm there is a significant reduction of seizure durations. When at hypothalamus stimulation instead of theta rhythm the electrical activity is desynchronized, there occurs a considerable intensification of seizure activity. Therefore, seizure-theta antagonism in our experiments could be interpreted as an adjustment of the inhibitory mechanisms when the theta rhythm is evoked.
\end{abstract}

\section{Keywords}

Hippocampus, Seizure, Theta Rhythm, Rats 


\section{Introduction}

At present, a number of forms of epileptic attacks are intractable (not sensitive to pharmacological treatment) [1]. The search for alternative possibilities for therapy of such disorders motivated ones to study such "antiseizure" approaches as electrical stimulation of afferent nerves and/or profound structures of the brain. Stimulations of the cerebellum, locus coeruleus, solitary tract nucleus, as well as of the nerve vagus were tested as influences potentially capable of blocking seizure attacks in humans and experimentally evoked epileptiform discharges in animals [2] [3] [4]. The results of using such methods were, however, contradictory. This is due to insufficient knowledge of the functional organization of the structures, disorders in which result in the development of epilepsy, and different forms and models of epilepsy used in the above-cited studies. This is why structures whose stimulation is capable of inducing an antiseizure effect by preventing initiation and/or spreading of epileptiform reactions have still not been adequately identified.

Investigations of a number of authors have shown that the hippocampus generates rhythmical slow activity, or theta rhythm, in response to stimulation of various receptors, as well as of direct stimulation of some of the brain structures, gross body movements and paradoxical sleep or emotional behavior [5]. Other behavioral states such as awake immobility and slow wave sleep are accompanied by large amplitude irregular activity [6]. Another important characteristic of the hippocampus is high susceptibility to epileptogenic electrical or chemical agents, and it has become evident that the hippocampus, and other limbic structures, are especially prone to develop a seizure activity [7] [8] [9] [10] [11].

It has been demonstrated by us that in the course of kindling, during simultaneous stimulation of the hippocampus and the dorsomedial nucleus of the hypothalamus (the structure whose activation causes reactions of fear, anxiety and run) there is a sharp halt in the development of epileptogenesis in the hippocampus. Also, in the presence of already developed epileptogenic focus in the hippocampus, combined stimulation of these structures resulted in suppression of the electroencephalographic seizure reactions and, respectively, manifestations of behavioral seizures reduced [12]. Our findings allow us to suppose that augmentation of inhibitory processes in hippocampal neurons in the course of dorsomedial hypothalamus (DMH) stimulation can trigger mechanisms preventing the development of epileptiform activity. Generation of the hippocampal $\theta$ rhythm is in fact a physiological state preventing the involvement of cerebral mechanisms in generalized seizure activity.

Because of two important characteristics of the hippocampus-theta rhythm and epileptogenesis-these appear to be interrelated in respect of their cellular substrates, and as far as theta rhythm may modulate hippocampal excitability, a study of the functional relationship between theta rhythm and seizure activity was started.

Therefore, reciprocal relations between the intensities of seizures and of $\theta$ ac- 
tivity [9] [13], on the one hand, and manifestations of seizures and the fear/anxiety state, on the other hand, can be interpreted as a sort of regulatory effects provided by inhibitory mechanisms under conditions of generation of the $\theta$ rhythm.

Hippocampal theta rhythm is a $3-12 \mathrm{~Hz}$ oscillation that, in rodents, occurs in wakefulness during exploration or other movements, and in a sustained fashion during paradoxical sleep or different emotional states. Hippocampal theta frequency activity is greatly reduced during spontaneous slow wave sleep [14] [15] [16]. These facts, and the critical role of the hippocampus in seizures, lead to the hypothesis that the theta rhythm is an indicator of a physiological state of the hippocampal formation which opposes its recruitment into seizures. The purpose of this study is to test this proposal by determining the effects on seizures of induction or suppression of hippocampal theta activity.

\section{Methods}

Wistar albino rats $(\mathrm{n}=32)$ weighing 250 to $300 \mathrm{~g}$ were kept under conditions of a 12/12 h illumination cycle with free access to food and water. Housing of, surgical manipulations with, and euthanasia of the animals were carried out in accordance with the rules and standards accepted by the scientific community of the European Union, legislation of Georgia, and the Committee on the care and use of animals in the Center of Life Sciences of Georgia (20.11.2019). Instructions of the administration of the National Institutes of Health (Bethesda, USA) on the care and use of laboratory animals (NIH Publication No. 88-2959) were also taken into account.

The animals were anesthetized by sodium pentobarbital $(40 \mathrm{mg} / \mathrm{kg}$, i.p.). Bipolar stimulating/recording electrodes (stainless steel) were stereotaxically [17] implanted bilaterally in the ventral hippocampus and DMH. After at least 10 - 12 days post-surgery rats were electrically stimulated in the hippocampus according to the kindling paradigm. Animals subjected to everyday 12 sessions of hippocampal stimulations with 30-min-long intervals in a 4-day-long session.

Experiment 1. During electrodes implantation to rats $(\mathrm{n}=10)$ and after the lapse of post-operation period (10 - 12 days) we studied the influence of strong (100 - $110 \mathrm{~dB})$ sound stimulation on the course of hippocampal stimulation induced local (without behavioral manifestations) seizure reactions. Sound stimulus was presented during $28-30 \mathrm{~s}$, while stimulation of hippocampus ( $40 \mu \mathrm{A}, 0.5$ ms, $80 \mathrm{~Hz}$ ) was applied during $10 \mathrm{~s}$ (see Figure 1).

Experiment 2. Emerging from our experience and knowing that in the course of kindling in rats by this scheme, there frequently occurs spontaneous electroencephalographic (EEG) and occasionally behavioral seizure development, therefore it was decided to study the course of EEG seizure reactions in different stages of sleep-wakefulness $(\mathrm{n}=10)$.

Experiment 3. After the lapse of post-operation period in rats $(\mathrm{n}=12)$ we studied the effect of hypothalamic stimulation, using different parameters, on the 


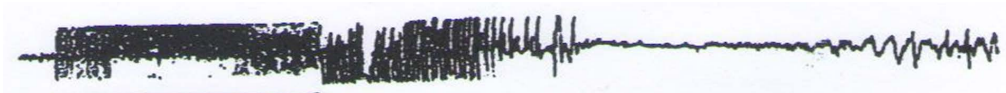

(a)

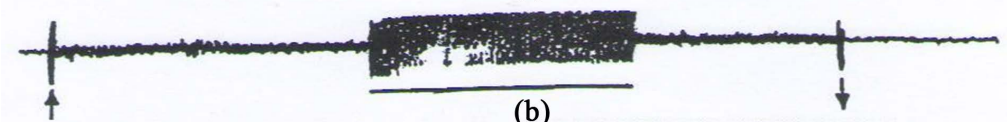

(b)

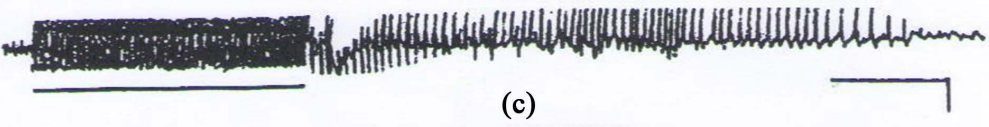

(c)

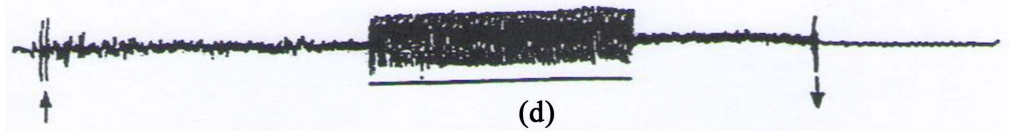

Figure 1. Influence of the strong audiogenic stimulation on the hippocampal electrographic seizure activity. (a) (c) Effects of contralateral hippocampal stimulation (40 $\mu \mathrm{A}$, $0.5 \mathrm{~ms}, 80 \mathrm{~Hz}$ ). (b) (d) Impact of acoustic irritation on the development of hippocampal seizures. Stimulation of the hippocampus is indicated by the horizontal line. The errows indicate on- and off-DMH stimulation. It should be noted that at a strong sound stimulation the theta rhythm cannot be seen on the EEG because of low amplification of the electroencephalograph during the development of high-amplitude seizure activity. Calibration-250 $\mu \mathrm{V}, 4$ Section.

course of hippocampal theta rhythm and seizure reactions. Electrodes were implanted in the dorsal hippocampus bilaterally. The stimulating electrode was implanted on one side, while the recording one contralaterally. The registration recording electrodes were four electrodes adhered to each other whose intertip distance was $100-150 \mu \mathrm{m}$.

After the kindling procedure, spontaneous (larval) seizure dischargers were found in 8 rats. The parameters of these seizure dischargers were examined within the sleep-wakefulness cycle and under conditions of emotional behavior. The field electrical activity of the hippocampus (electrohippocampogram, EHG) was recorded and processed using a 10-channel analyzer/integrator, ANJEG-81 (Medicor, Hungary). Characteristic frequencies of the $\delta$ and $\theta$ activities were equal, respectively, to 2.2 and $5.3 \mathrm{~Hz}$. The EEG and EHG were recorded using a multichannel inkwriter.

\section{Data Analysis}

Data analysis was performed with GNU PSPP software version 0.6.2 using the methods of descriptional statistics, paired 2-tailed t-test $(\alpha<0.05)$.

\section{Results}

As noted above, stimulation of the DMH resulted in significant suppression of both electrographic and behavioral manifestations of seizure activity. Stimulation of the DMH not only inhibited the development of generalized seizures in the process of epileptogenesis, but also significantly decreased the number and duration of generalized convulsions in rats with preliminarily evoked epileptic manifestations. There is no doubt about the fact that not only experimentally 
induced electrical stimulation of an emotiogenic structure, but also under the impact of strong unusual stimuli, existing in the environment, in animals (and in humans as well) observed are reactions of anxiety and fear. In illustration of this, we can mention the result of one experiment which shows the impact of strong sound stimulus (95-100 dB) on the development of local hippocampographic seizures induced by hippocampal stimulation (Figure 1). It is seen that against background of strong sound stimulation occurred blockade of local seizure reactions induced by hippocampal stimulation.

Epileptic seizures frequently develop within the sleep periods [14] [16]. In our earlier investigations changes in seizure activity of brain caused by electrical stimulation of the hippocampus were investigated at various sleep and wakefulness stages. It was shown that during slow sleep, when high voltage slow waves are predominant in the electroencephalogram, near-threshold epileptogenic hippocampal stimulation caused pronounced paroxismal discharges. During wakefulness or paradoxical phase of sleep, when EEG is desynchronized and when theta rhythm arises in hippocampus, stimulation with the same parameters proved to be less effective: seizure discharges either are weakened or do not emerge at all [18].

As was mentioned above, a noticeable part of the experimental rats $(\mathrm{n}=8)$ demonstrated spontaneous abortive seizure discharges in the composition of hippocampal electrical activity after realization of the fast kindling paradigm. Criteria for classification of the sleep/wakefulness states were based on results of recording of changes in the amplitude and frequency of hippocampal electrical activity and in externally observed behavior. Before the beginning of kindling stimulation of the hippocampus, when the animal appeared to be in the awake state with no manifestations of emotions, the hippocampal activity mostly included fast oscillations. Vice versa, when the rat was in the state of quiet sleep in a comfortable position, slow/high-amplitude waves were observed in electrical activity of the hippocampus. Rather specific changes in hippocampal electrical activity (EHG) were manifested within periods of paradoxical sleep. In these intervals, highly regular $\theta$ waves were observed.

After the kindling process terminated and also after 8 to 9 days, larval interictal epileptiform activity was noticed in the EHG composition of fully awake rats. The interictal epileptiform activity within different phases was modified in a typical way. The frequency and amplitude of these discharges increased immediately with the transition from the awake state to drowsiness and a slow-wave sleep phase. After the animal came from slow-wave sleep to paradoxical sleep, epileptiform activity in the EHG composition completely disappeared (Figure 2).

It is noteworthy that since the third day of hippocampal stimulation, the electrographic and bevavioral seizure reactions were followed with fear, anxiety, and other active behavioral reactions, which continued for $1-2$ min after cessation of the electrographic discharges. Along with these reactions, the hippocampal 


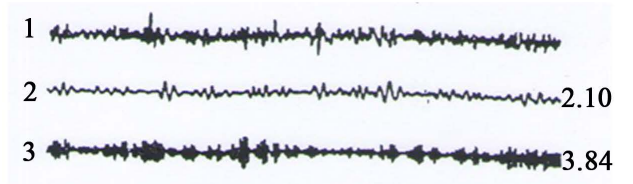

(a)

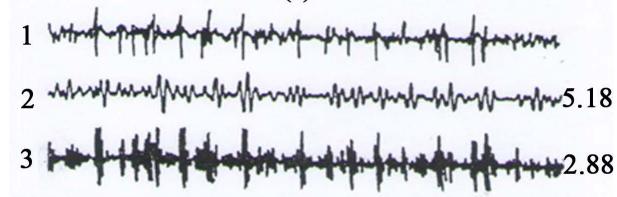

(b)

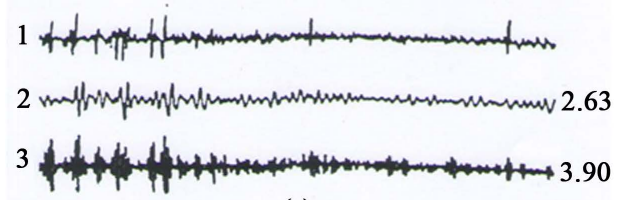

(c)

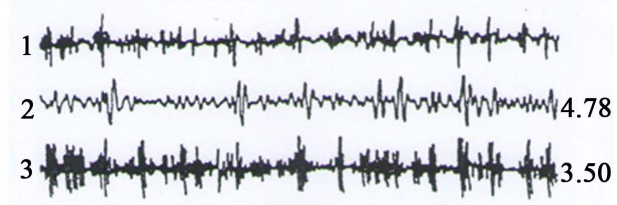

(d)

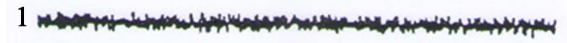

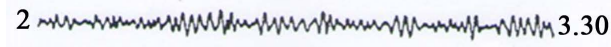

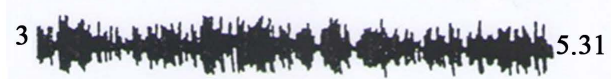

(e)

$$
\underset{1 \mathrm{sec}}{\mathrm{L}} 250 \mu \mathrm{V}
$$

Figure 2. EHG after the kindling procedure. (a) Records in the awake state; (b) (d) Those in the slow-wave sleep state; (e) Those during paradoxical sleep; (c) Transition of slow-wave sleep to paradoxal sleep. Traces 1 are native EHGs, traces 2 and 3 are differentiated $\delta$ and $\theta$ components, respectively; powers of oscillations of the respective frequencies, arb. units, are shown on the right.

theta activity did develope. This period is not characterized with the spontaneous epileptic discharge development within the inter-stimulation time. As soon as the animal transited into the rest and/or drowsy state, a sponpaneous epileptic discharge did occur (unpublished data).

It is remarkable that in the case of stimulation of the hypothalamus as well as of the brainstem reticular formation, change in the electrical activity of the hippocampus is dependent on the stimulation parameters. Consequently these structures have a diverse action on the development of seizure reactions in the hippocampus.

In experiments we have examined the effects of experimental induction and/or suppression of the hippocampal theta activity on the evoked hippocampal seizures (Figure 3 and Figure 4).

Figure 3 depicts durations of local hippocampal eightfold single stimulation induced seizure reactions against the background of stimulation of isolated 


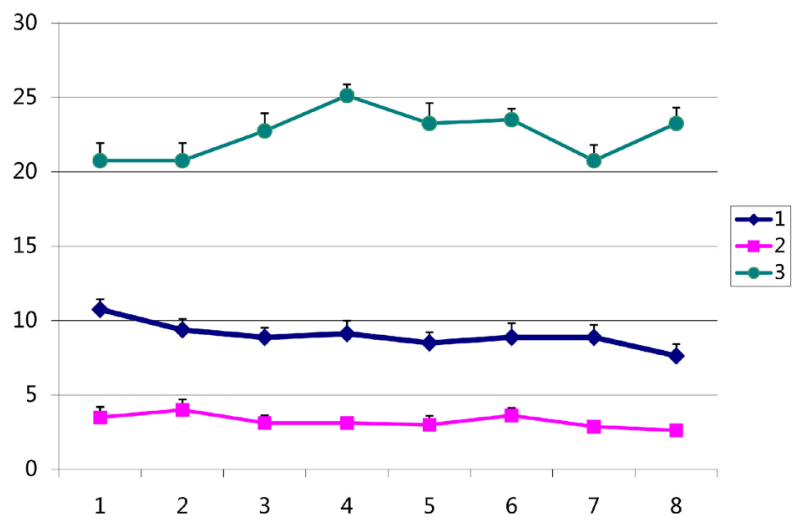

Figure 3. Influence of stimulation with different parameters of DMH on the course of hippocampal seizures. 1-Contralateral hippocampal seizures induced by single irritation (50 $\mu \mathrm{A}, 0.3 \mathrm{~ms}) .2$-Contralateral hippocampal seizures induced by stimulation with the same parameters on the background hypothalamic threshold stimulation ( $30 \mu \mathrm{A}, 0.3 \mathrm{~ms})$. 3-Contralateral hippocampal seizures induced by stimulation with the same parameters on the beckground of subtreshold stimulation of the hypothalamus ( $60 \mu \mathrm{A}, 0.3 \mathrm{~ms})$. Ordinate-duration of seizures, min; Abscissa-number of animals.

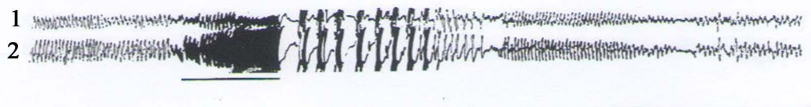

(a)

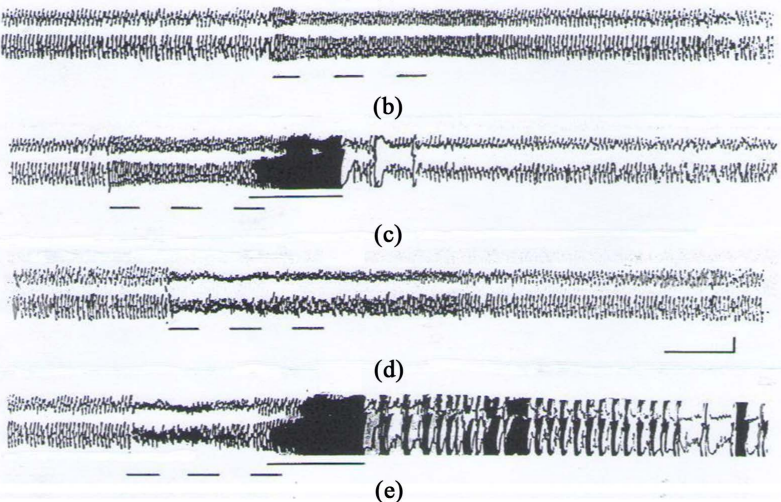

Figure 4. Influence of prior hypothalamic stimulation on the evoked seizure activity. (a) Effects of contralateral hipocampal stimulation $(30 \mu \mathrm{A}, 0.3 \mathrm{~ms}, 60 \mathrm{~Hz})$; (b) Effects of DMH threshold stimulation $(25 \mu \mathrm{A}, 0.3 \mathrm{~ms}, 80 \mathrm{~Hz}$ ); (c) Effects of preliminary hypothalamic threshold stimulation on the seizure activity; (d) Effects of DMH suprathreshold stimulation $(40 \mu \mathrm{A}, 0.3 \mathrm{mc}, 80 \mathrm{~Hz})$; (e) Effects of preliminary hypothalamic suprathreshold stimulation on the seizure activity. Stimulation of the hippocampus is indicated by the horizontal line. The bold line indicates stimulation of DMH. Calibration $-250 \mu \mathrm{V}, 3$ Section.

hippocampus (1), and DMH with threshold (2) and suprathreshold (3) intensities. It is seen that at threshold stimulation of hypothalamus when electrohippocampogram shows augmentation of the theta rhythm there is a significant reduction of seizure durations. Vice versa, when at hypothalamus stimulation instead of theta rhythm the electrical activity is desynchronized, there occurs a considerable intensification of seizure activity (see Figure 4). 


\section{Discussion}

Several findings indicate that activation of septo-hippocampal pathways by sensory, hypothalamic and brainstem stimulations was accompanied by a decrease in the discharge frequency of hippocampal pyramidal cells [19]. Interneurons, on the other hand, were found to increase their firing rate. Also, during paradoxical sleep the spontaneous activity of pyramidal cells was found to be lowest, accompanied by most regular theta waves [20] [21].

Most numerous among the hippocampal interneurons are the basket cells and their functional role is inhibition. The interneurons contain glutamic acid decarboxylase, the synthesized enzyme for the inhibitory neurotransmmiter-gamma-aminobutyric acid (GABA). The epileptic activity suggests that seizures are generated by an enhancement of the excitatory phenomena [22] [23] associated with a lack of inhibitory function, particularly that of GABA-mediated activity. Decreased inhibition seems to occur through competitive blocking during seizure activity preventing the enhancement of the membrane conductance evoked by GABA. The hippocampal interneurons are considered to be theta cells [24] [25]. It is known that there is a drastic increase in firing of such cells when the Theta rhythm is evoked, indicating augmented GABA release.

Karunakaran et al. [11] studied the role of hippocampal CA3 interneurons at Pilocarpine induced seizure activity. It turned out that theta-on interneurons selectively increase their firing rate at seizure onset. It is known that in the patients suffering from temporal lobe epilepsy interictal emotional disorders are seen. This fact appears particularly interesting while interpreting our evidence. In the experiments [12] where the influence of hypothalamic stimulation on kindling development was studied it was shown that stimulation of this structure in question significantly suppressed the course of both EEG and behavioral seizure reactions. Then it was thought that stimulation of emotiogenic structures, as well as enhancement of emotional reactions must interictally condition triggering of the mechanisms which cause seizure inhibition. Among one of these mechanisms attention was focused on the appearance of theta rhythmin parallel to development of emotional reactions. Appearance of theta rhythm prior to seizure onset, also emotional disorders in humans some hours earlier [23], in our opinion, must indicate coming into play of endogenous antiepileptic mechanisms.

It is clear that triggering of possible antiepileptic mechanisms often fails to block seizure reactions, but the existence of such mechanisms may assist neurologists not to use in the epileptic patients the antiepileptic drugs which cause reduction of interictal emotional reactions.

Emerging from our experiments we can conclude that the emotional disturbances can be considered as the emergence of instinctive behavior with an adaptive significance of defence and as a by-product of the inhibitory processes that build-up to protect against the future occurrence of seizures, that gives support to our assumption that in Karunakarans et al., studies activation of theta interneurons may reflect recruitment of those mechanisms which suppress seizure development. This should be similar to the data where showing in epileptic pa- 
tients, emotional disorders develop 1 - 2 day and especially 24 hours before the onset of seizures [23], or Xiaoxuan et al. findings likewise hold interest showing that interictal spikes have a significant negative impact on theta rhythm in human temporal lobe epilepsy [10].

Therefore, seizure-Theta antagonism in our experiments could be interpreted as an adjustment of the inhibitory mechanisms when the theta rhythm is evoked. It is assumed that increased inhibition during the hippocampal theta activity may trigger the mechanisms preventing the epileptiform activity and that hippocampal theta rhythm is a physiological state of the hippocampus, which opposes its recruitment into seizures.

Several lines of evidence suggest that activation of emotiogenic structures and brainstem reticular formation hampers the emergence of limbic seizure discharges [26]. Although it was shown that in the case of stimulation of the hypothalamus as well as of the brainstem reticular formation, changes in the electrical activity of the hippocampus is dependent on the stimulation parameters. The threshold stimulation of hypothalamus when EHG shows augmentation of the theta rhythm there is a significant reduction of seizure durations. But when at hypothalamus stimulation instead of theta rhythm the electrical activity is desynchronized, there occurs a considerable intensification of seizure activity. This must not be surprising, for any frequency synchronous electrical activity of the brain (as well as of theta rhythm) implies enhancement of inhibitory processes (implied is the rhythmicity of inhibitory postsynaptic potentials). During desynchronization of electrical activity occurs blockade of the inhibitory potentials (as was in our case at suprathreshold stimulation of hypothalamus). This mechanism must underlie in one case diminution of seizure reactions at the stimulation of hypothalamus using different parameters (upon enhancement of theta rhythm) and in the other case, its enhancement (desynchronization of EHG).

Except this possibility can be assumed that the intensification of seizure activity by the suprathreshold stimulation of hypothalamus may be determined, on the one hand, by its facilitatory influence on the thalamo-cortical system [27], and also, by suppressing impact on the diencephalic inhibitory structures-mostly on the thalamic reticular nucleus [28] on the other hand.

\section{Acknowledgements}

The research was conducted in frames of Petre Shotadze Tbilisi Medical academy. Funding program for development and support of Scientific Research Projects. The authors thank Ms. Ninelli Skhirtladze for her help in the preparation of the manuscript.

\section{Conflicts of Interest}

The authors declare no conflicts of interest regarding the publication of this paper.

\section{References}

[1] Forsgen, L. (1995) Epidemiology of Intractable Epilepsy in Adults. In: Johannessen, 
S.I., Gram, L., Sillanpau, M. and Tomson, T., Eds., Intractable Epilepsy, Wrightson Biomedical Publishing Ltd., Petersfield, 25-40.

[2] Davis, R. (2000) Cerebellar Stimulation for Cerebral Palsy Spasticity, Function and Seizures. Archives of Medical Research, 31, 290-299.

https://doi.org/10.1016/S0188-4409(00)00065-5

[3] Magdaleno-Madrigal, V., Valdes-Cruz, A. and Martinez-Vargas, D. (2002) Effect of Electrical Stimulation of the Nucleus of the Solitary Tract on the Development of Electrical Kindling in the Cat. Epilepsia, 43, 964-969.

https://doi.org/10.1046/j.1528-1157.2002.05702.x

[4] Chanel, S., Westerveld, M. and Spenser, S. (2003) Long-Term Outcome of Vagus Nerve Stimulation for Refractory Partial Epilepsy. Epilepsy \& Behavior, 4, 302-309. https://doi.org/10.1016/S1525-5050(03)00109-4

[5] Vanderwolf, C.H., Kramis, R. and Robinson, T.E. (1978) Hippocampal Electrical Activity during Waking Behavior and Sleep: Analyses Using Centrally Acting Drugs. In: Elliot, K. and Whelan, J., Eds., Functions of the Septo-Hippocampal System, Ciba Foundation Symposium, 58, Elsevier, Amsterdam, 199-221.

[6] Buzsáki, G., Buhl, D.L., Harris, K.D., Csicsvari, J., Czéh, B. and Morozov, A. (2003) Hippocampal Network Patterns of Activity in the Mouse. Neuroscience, 116, 201-211. https://doi.org/10.1016/S0306-4522(02)00669-3

[7] Liberson, W.T. and Akert, K. (1955) Hippocampal Seizure States in Guinea Pig. Electroencephalography and Clinical Neurophysiology, 7, 211-222.

https://doi.org/10.1016/0013-4694(55)90036-5

[8] Green, J.D. and Shimamoto, T. (1953) Hippocampal Seizure End Their Propagation. Archives of Neurology and Psychiatry, 70, 687-702. https://doi.org/10.1001/archneurpsyc.1953.02320360002001

[9] Toyoda, I., Fujita, S., Thamattoor, A.S. and XPaul Buckmaster, P.S. (2015) Unit Activity of Hippocampal Interneurons before Spontaneous Seizures in an Animal Model of Temporal Lobe Epilepsy. The Journal of Neuroscience, 35, 6600-6618. https://doi.org/10.1523/JNEUROSCI.4786-14.2015

[10] Fu, X.X., Wang, Y.H., Ge, M.L., Wang, D.H., Gao, R.G., Wang, L., Guo, J.D. and Liu, H.S. (2018) Negative Effects of Interictal Spikes on Theta Rhythm in Human Temporal Lobe Epilepsy. Epilepsy and Behavior, 87, 207-212. https://doi.org/10.1016/j.yebeh.2018.07.014

[11] Karunakaran, S., Grasse, D.W. and Moxon, K.A. (2016) Role of CA3 Theta-Modulated Interneurons during the Transition to Spontaneous Seizures. Experimental Neurology, 283, 341-352. https://doi.org/10.1016/j.expneurol.2016.06.027

[12] Nanobashvili, Z.I., Bilanishvili, I.G., Barbakadze, M.G. and Khizanishvili, N.A. (2019) Mood Disorders: Protection of the Hyper-Excited Brain, or a Risk Factor? Journal of Behavioral and Brain Science, 9, 54-65. https://doi.org/10.4236/jbbs.2019.92005

[13] Kilias, A., Häussler, U., Heining, K., Froriep, U., Haas, C.A. and Egert, U. (2018) Theta Frequency Decreases throughout the Hippocampal Formation in a Focal Epilepsy Model. Hippocampus, 6, 375-391. https://doi.org/10.1002/hipo.22838

[14] Frauscher, B. and Gotman, J. (2019) Sleep, Oscillations, Interictal Discharges, and Seizures in Human Focal Epilepsy. Neurobiology of Disease, 127, 545-553. https://doi.org/10.1016/j.nbd.2019.04.007

[15] Licchetta, L., Vignatelli, L., Zenesini, C., Mostacci, B., Ferri, L., Provini, F., Tinuper, P. and Bisulli, F. (2019) Sleep-Related Hypermotor Epilepsy: A Prediction Cohort Study on Sleep/Awake Patterns of Seizures. Epilepsia, 60, e115-e120. 
https://doi.org/10.1111/epi.16369

[16] Jain, S.V. and Kothare, S.V. (2015) Sleep and Epilepsy. Seminars in Pediatric Neurology, 2, 86-92. https://doi.org/10.1016/j.spen.2015.03.005

[17] Paxinos, G. and Watson, C. (1997) The Rat Brain in Stereotaxic Coordinates. Academic Press, New York.

[18] Ioseliani, T.K., Nanobashvili, Z.I. and Khizanishvili, N.A. (1974) Threshold Fluctuation of Seizure Activity of Cat Brain in Various Stages of Sleep and Wakefulness. Neirofiziologia, 6, 577-584. (In Russian)

[19] Euler, V. and Green, J.D. (1960) Excitation, Inhibition and Rhythmical Activity in Hippocampal Pyramidal Cells in Rabbits. Acta Physiologica Scandinavica, 48, 110-125. https://doi.org/10.1111/j.1748-1716.1960.tb01851.x

[20] Delacour, J. (1980) Conditioned Modifications of Arousal and Unit Activity in the Rat Hippocampus. Experimental Brain Research, 38, 95-101. https://doi.org/10.1007/BF00237935

[21] Mays, L.E. and Best, P. (1975) Hippocampal Unit Activity to Total Stimuli during Arousal from Sleep and in Awake Rats. Experimental Neurology, 47, 268-279. https://doi.org/10.1016/0014-4886(75)90256-3

[22] Dingledine, R. and Gjerstad, L. (1980) Reduced Inhibition during Epileptiform Activity in the in Vitro Hippocampal Slice. Journal of Physiology (London), 305, 297-313. https://doi.org/10.1113/jphysiol.1980.sp013364

[23] Kanner, A.M. (2003) Depression in Epilepsy: Prevalence, Clinical Semiology, Pathogenic Mechanisms, and Treatment. Biological Psychiatry, 54, 388-398. https://doi.org/10.1016/S0006-3223(03)00469-4

[24] Buzsaki, G. and Eidelberg, E. (1982) Direct Afferent Excitation and Long-Term Potentiation of Hippocampal Interneurons. Journal of Neurophysiology, 48, 397-607. https://doi.org/10.1152/jn.1982.48.3.597

[25] Freund, T.F. and Buzsaki, G. (1996) Interneurons of the Hippocampus. Hippocampus, 6, 347-470. https://doi.org/10.1002/(SICI)1098-1063(1996)6:4<347::AID-HIPO1>3.0.CO;2-I

[26] Guerrero-Figueroa, R., Lester, B. and Heath, R.G. (1965) Changes of Hippocampal Epileptiform Activity during Wakefulness and Sleep. Acta Neurológica Latinoamericana, 11, 330-349.

[27] Herrera, C.G., Cadavieco, M.C., Jego, S., Ponomarenko, A., Korotkova, T. and Adamantidis, A. (2016) Hypothalamic Feedforward Inhibition of Thalamocortical Network Controls Arousal and Consciousness. Nature Neuroscience, 19, 290-298. https://doi.org/10.1038/nn.4209

[28] Nanobashvili, Z.I., Surmava, A.G., Bilanishvili, I.G., Barbaqadze, M.G., Mariamidze, M.D. and Khizanishvili, N.A. (2012) Significance of the Thalamic Reticular Nucleus GABAergic Neurons in Normal and Pathological Activity of the Brain. Journal of Behavioral and Brain Science, 2, 436-444. https://doi.org/10.4236/jbbs.2012.24051 